\title{
Spin-orbit driven electrical manipulation of the zero-field splitting in high-spin centers in solids
}

\author{
Timur Biktagirov (1) and Uwe Gerstmann (1) \\ Lehrstuhl für Theoretische Materialphysik, Universität Paderborn, 33098 Paderborn, Germany
}

(Received 5 September 2019; accepted 24 March 2020; published 23 April 2020)

\begin{abstract}
In recent years, spin-orbit coupling has attracted significant attention due to its promising applications in spintronic devices. In solid-state spin qubits, the spin-orbit coupling allows for the lifting of spin degeneracy in the absence of an external magnetic field. Such spin-orbit driven zero-field splitting can be directly tuned by external electric fields. Here we present a reliable theoretical framework to address this phenomenon in extended periodic systems. We unravel the microscopic origin of the zero-field splitting in light-element semiconductors and propose its implications for coherent electrical control. The reported theoretical results open up promising possibilities for a rational design and tuning of high-spin centers suitable for quantum information processing.
\end{abstract}

DOI: 10.1103/PhysRevResearch.2.023071

\section{INTRODUCTION}

Defects in the solid state are attracting tremendous attention as promising tools for scalable quantum computing [1]. As a part of a multiqubit quantum computer, the defects must be both coherent and easy to control. A promising strategy to achieve this is by coupling their electron spin to external electric fields via inducing Stark shifts of, for example, their $g$ factors or electron-nuclear hyperfine couplings [2-7]. Thereby, the desired qubits can be selectively brought into resonance with the global microwave field and subjected to quantum operations [8]. The technological benefit of such a protocol is that oscillating electric fields are easy to generate and apply with precise spatial selectivity via nanoscale electrodes.

For high-spin $(S \geqslant 1)$ qubits, an attractive target for the Stark-shift control is the energy splitting between the $\left|m_{S}\right\rangle$ spin states of high-spin qubits due to magnetic anisotropy in the absence of an external magnetic field [cf. Fig. 1(a)] $[9,10]$. The electrical tuning of this zero-field splitting (ZFS) has been demonstrated experimentally [11-15], but the physical origin of the E-field dependence remains debatable. A qualitative explanation can be found in perturbation theory. In first order, the ZFS stems from the spatial anisotropy of the magnetization density [so-called spin-spin (SS) contribution]. At the same time, second-order perturbation theory provides the dependence of ZFS on the spin-orbit (SO) coupling. An external electric field distorts the spin density of the qubit and thereby alters its angular momentum. Thus, it can affect both the SS and SO part of the ZFS. The SO-driven ZFS can directly experience the E-field dependence via an external

Published by the American Physical Society under the terms of the Creative Commons Attribution 4.0 International license. Further distribution of this work must maintain attribution to the author(s) and the published article's title, journal citation, and DOI. contribution to the SO coupling Hamiltonian

$$
\hat{\mathcal{H}}^{\mathrm{SO}}(\mathbf{E})=\frac{\alpha^{2}}{4} \hat{\sigma} \cdot[\nabla V(\mathbf{r})-\mathbf{E}] \times \hat{\mathbf{p}} .
$$

Similar to the well-known Rashba effect [16,17], this allows manipulating the spin without the aid of a magnetic field.

While the SS ZFS of spin qubits has recently become tractable with density functional theory (DFT) [18-22], theoretical rationalization of the SO-driven contribution has been so far limited to finite-size molecular systems [23-26]. The aim of this work is thus to present an efficient and accurate method to address the missing SO part for high-spin states in extended periodic systems and explore its implication for electrical control.

\section{METHOD AND VALIDATION}

In order to model a solid-state qubits, we use the conventional supercell approach in combination with the computationally efficient pseudopotential approximation. When complemented by the projector augmented wave (PAW) method [27], this strategy allows all-electron accuracy not only for total energies and forces, but also for the calculation of magnetic coupling parameters, including the SS ZFS [19]. Since many of today's candidates for quantum technological applications exhibit relatively small ZFS in the range of some $\mathrm{MHz}$, we develop and implement a perturbative treatment of its SO part within a PAW framework. We validate our approach for the case of large SO ZFS when it can be addressed directly via fully relativistic total energy differences. Finally, by considering a family of high-spin impurity-vacancy defects in diamond, we rationalize the origin of the SO-driven magnetic anisotropy in light-element materials and explore its amenability to electrical control.

In order to address the relation between magnetic anisotropy and ZFS, we can include $\mathcal{H}^{\mathrm{SO}}$ directly into the Kohn-Sham equations, perform relativistic self-consistent calculations, and, thereby, evaluate the total energy of the system as a function of the spin quantization direction, $E^{\text {tot }}\left(\vec{n}_{\sigma}\right)$. In a uniaxial case, the magnetic anisotropy energy is then 
(a)
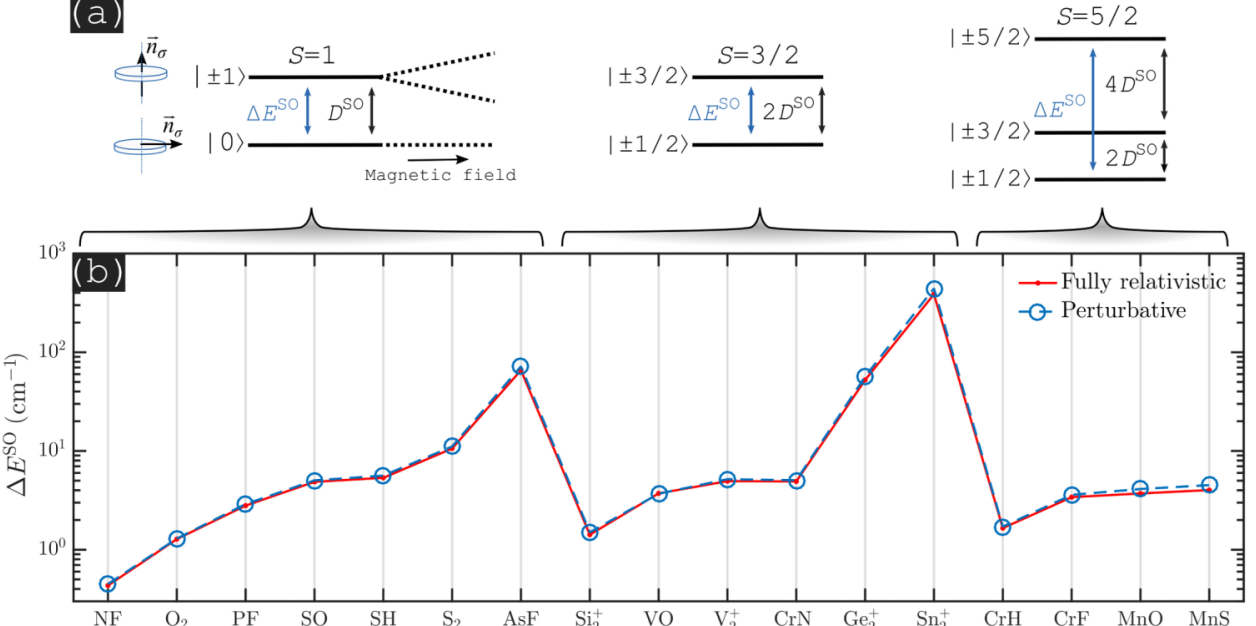

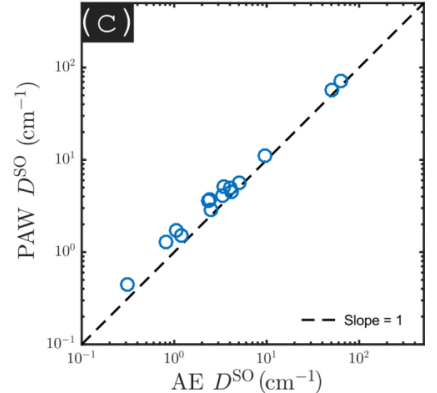

FIG. 1. Validation of the perturbative PAW-based implementation of SO ZFS for a set of diatomic radicals. (a) Energy level diagrams of high-spin systems illustrating the connection between spin reorientation barrier, $\Delta E^{\mathrm{SO}}$, and the zero-magnetic field splitting of its $\left|m_{S}\right\rangle$ spin sublevels ( $D^{\mathrm{SS}}$ omitted). (b) $\Delta E^{\mathrm{SO}}$ values calculated fully relativistically according to Eq. (2) (red line) and via the perturbative approach (blue circles). (c) Comparison of the $D^{\mathrm{SO}}$ values obtained with the PAW approach (this work) and an all-electron (AE) method (ORCA software [40], PBE functional, and def2-TZVP basis set [41]).

defined as a difference between $E^{\text {tot }}\left(\vec{n}_{\sigma}\right)$ obtained with $\vec{n}_{\sigma}$ perpendicular and parallel to the anisotropy direction:

$$
E^{\mathrm{SO}}=E^{\mathrm{tot}}(\|)-E^{\mathrm{tot}}(\perp) \text {. }
$$

In magnetic resonance spectroscopy, the resulting ZFS within the $\left|m_{S}\right\rangle$ multiplet is conventionally characterized by the so-called $D$ value [cf. energy level diagrams in Fig. 1(a)]. It is related to the magnetic anisotropy energy via a specific total-spin $(S)$-dependent prefactor [10]:

$$
\begin{aligned}
& D^{\mathrm{SO}}=\Delta E^{\mathrm{SO}} / S^{2} \text { for integer } S, \\
& D^{\mathrm{SO}}=\Delta E^{\mathrm{SO}} /\left(S^{2}-1 / 4\right) \text { for-half integer } S .
\end{aligned}
$$

This direct, total-energy difference approach was applied to a benchmarking set of high-spin $(S=1,3 / 2$ and $5 / 2)$ diatomic radicals with uniaxial magnetic anisotropy [see Fig. 1(b)]. Thereby, we profit from our previous implementation [28] in the QUANTUM ESPRESSO software [29,30], which allows for accurate relativistic total energy calculations based on standard (scalar relativistic) pseudopotentials even for systems with giant SO coupling [28]. The PBE functional [31] was adopted to describe exchange-correlation energy. We used norm-conserving pseudopotentials [32] with two PAW projectors per angular momentum channel together with a plane-wave (PW) basis set with a kinetic energy cutoff up to $100 \mathrm{Ry}$. In order to impose periodic boundary conditions, the molecules were immersed into a cubic box of $15^{3} \AA^{3}$.

The presented strategy exhibits brilliant numerical stability for finite-size molecules with relatively large $\Delta E^{\mathrm{SO}}$ (compared to the total energy). On the other hand, we do not expect it to be generally applicable to solid-state defects with small magnetic anisotropy embedded in large supercells. Direct calculation of $\Delta E^{\mathrm{SO}}$ via total energy differences in the $10^{-8} \mathrm{eV}$ regime and below might require prohibitively accurate convergence. For this reason, we develop a more versatile alternative, i.e., a perturbative approach. It accomplishes our previous work on spin-spin ZFS [19] and, thus, has been equally implemented in the GIPAW-tree of the QUANTUM ESPRESSO package [29]. We follow the basic ideas presented in Refs. [33-35] for finite systems and make them applicable for periodic systems while using the PAW pseudopotential framework. On this level, the SO coupling Hamiltonian reads $[28,36]$

$$
\hat{\mathcal{H}}^{\mathrm{SO}}=\frac{\alpha^{2}}{4}\left[\hat{\sigma} \cdot \nabla V_{\mathrm{ps}}(\mathbf{r}) \times \hat{\mathbf{p}}+\sum_{\mathbf{R}, i, j}\left|p_{\mathbf{R}, i}\right\rangle \hat{\sigma} \cdot \mathbf{f}_{\mathbf{R}, i j}\left\langle p_{\mathbf{R}, j}\right|\right] .
$$

$V_{\mathrm{ps}}(\mathbf{r})$ is the local part of the pseudopotential, and $\left|p_{\mathbf{R}, i}\right\rangle$ are the PAW projector functions being nonzero only within the augmentation spheres around each atom $\mathbf{R}$. The matrix elements $\mathbf{f}_{\mathbf{R}, i j}$ depend on the spherically symmetric all-electron and pseudopotentials, $\mathcal{V}_{\mathrm{ae}}(r)$ and $\overline{\mathcal{V}}_{\mathrm{ps}}(r)$, both defined for each augmentation region [37]:

$$
\mathbf{f}_{\mathbf{R}, i j}=\left\langle\phi_{\mathbf{R}, i}\left|\frac{\hat{\mathbf{L}}}{r} \frac{\partial \mathcal{V}_{\mathrm{ae}}(r)}{\partial r}\right| \phi_{\mathbf{R}, j}\right\rangle-\left\langle\bar{\phi}_{\mathbf{R}, i}\left|\frac{\hat{\mathbf{L}}}{r} \frac{\partial \overline{\mathcal{V}}_{\mathrm{ps}}(r)}{\partial r}\right| \bar{\phi}_{\mathbf{R}, j}\right\rangle
$$

where $\left|\phi_{\mathbf{R}, i}\right\rangle$ and $\left|\bar{\phi}_{\mathbf{R}, i}\right\rangle$ are all-electron and pseudopartial waves for atom $\mathbf{R}$ and quantum numbers $l_{i}$ and $m_{i}$.

As already evaluated in Ref. [36] for the orbital magnetization, we redefine the SO coupling for collinear spin polarization, i.e., by substituting the spin operator $\hat{\sigma}$ in Eq. (4) with $\hat{\sigma}_{a}=\left(\begin{array}{cc}1 & 0 \\ 0 & -1\end{array}\right) \cdot \mathbf{e}_{a}$ (with $a=x, y, z$ ). Then the SO coupling in direction $a\left(\hat{\overline{\mathcal{H}}}_{a}^{\text {sO }}\right)$ and $b\left(\hat{\mathcal{\mathcal { H }}}_{b}^{\text {so }}\right)$ contributes to the total energy of the system in second-order perturbation theory as

$$
E_{a b}^{\mathrm{SO}}=\sum_{o, s, s^{\prime}} \operatorname{Re}\left\langle\bar{\Psi}_{o}^{s}\left|\hat{\overline{\mathcal{H}}}_{a}^{\mathrm{SO}} \mathcal{G}^{s^{\prime}}\left(\epsilon_{o}\right) \hat{\overline{\mathcal{H}}}_{b}^{\mathrm{SO}}\right| \bar{\Psi}_{o}^{s}\right\rangle .
$$

Here the sum runs over the spin channels $s$ and $s^{\prime}$ and the occupied states $o \in s$. Thereby, $\left|\bar{\Psi}_{o}^{s}\right\rangle$ are the corresponding unperturbed Kohn-Sham wave functions (obtained without SO coupling), and $\mathcal{G}^{s^{\prime}}(\epsilon)$ is the Green's function of the empty 
TABLE I. Spin-orbit and spin-spin $[19,20]$ contributions to the ZFS (in $\mathrm{cm}^{-1}$ ) of the $\mathrm{NV}^{-}$center and a set of split-vacancy defects in diamond (compared to available experimental data).

\begin{tabular}{llllc}
\hline \hline Defect & $D^{\text {SO }}$ & \multicolumn{1}{c}{$D^{\text {SS }}$} & $D^{\text {SO+SS }}$ & Experiment \\
\hline $\mathrm{NV}^{-}$ & 0.0002 & 0.0908 & 0.0910 & $0.096^{\mathrm{a}}$ \\
$\mathrm{SiV}^{0}$ & 0.016 & 0.019 & 0.035 & $0.031^{\mathrm{b}}$ \\
$\mathrm{GeV}^{0}$ & 0.049 & 0.021 & 0.070 & $0.075^{\mathrm{c}}$ \\
$\mathrm{SnV}^{0}$ & 0.359 & 0.021 & 0.380 & \\
$\mathrm{PbV}^{0}$ & 4.832 & 0.022 & 4.854 & \\
\hline
\end{tabular}

${ }^{\mathrm{a}}$ Ref. [42].

${ }^{\mathrm{b}}$ Ref. [46].

${ }^{\mathrm{c}}$ Ref. [47].

states $e \in s^{\prime}$. In the current implementation, explicit summation over empty states is avoided by calculating $\mathcal{G}^{s^{\prime}}(\epsilon)$ according to the procedure described in Appendix A of Ref. [38] (i.e., by projecting the empty states onto the valence bands). By this, the approach becomes faster, numerically more stable, and almost unaffected by the underestimates band gap problem typically observed if using semilocal XC functionals.

The perturbative approach can be easily applied beyond the uniaxial case and for arbitrary orientation of the magnetic anisotropy direction within the cartesian frame. Indeed, the anisotropy energy is then defined by the principal values of the $3 \times 3$ matrix built according to Eq. (6): $\Delta E^{\mathrm{SO}}=E_{z z}^{\mathrm{SO}}-$ $\frac{1}{2}\left(E_{x x}^{\mathrm{SO}}+E_{y y}^{\mathrm{SO}}\right)$. As a consequence, the matrix elements $E_{a b}^{\mathrm{SO}}$ correspond directly to the elements of the SO part of conventional ZFS tensor, $\mathbf{D}^{\text {SO }}$ [39]. The resulting values for the molecular benchmarking set are plotted in Fig. 1(b) alongside the fully relativistic ones. Perfect agreement between the two approaches within a very broad range of the $\Delta E^{\mathrm{SO}}$ values covered by the benchmarking tests vividly demonstrates the validity of our perturbative treatment.

The finite size of the test systems also allows for direct comparison with an all-electron version of the perturbative approach [35] as available by the ORCA software package [40]. As illustrated in Fig. 1(c), our results reproduce the allelectron $D^{\mathrm{SO}}$ values nearly perfectly. Thereby, we demonstrate robustness of the adopted PAW pseudopotential formalism even for light elements. This allows us to further apply our perturbative treatment for high-spin solid-state defects.

\section{APPLICATION TO SOLID-STATE SPIN QUBITS}

We chose diamond as a host material because it remains one of the most promising platforms for defect-based quantum technologies. Modeling was done in periodically repeated cubic supercells (512 atoms) using a shifted $2 \times 2 \times 2 k$ point sampling. First, we confirm that the well-known and widely used negatively charged nitrogen vacancy center, $\mathrm{NV}^{-}$ [42], exhibits a small, almost negligible $D^{\text {SO }}$ value (about $10^{-4} \mathrm{~cm}^{-1}$, i.e., $D^{\mathrm{SO}} / \mathrm{h}$ of $5 \mathrm{MHz}$ ) in comparison to the spin-spin part of $0.091 \mathrm{~cm}^{-1}$ (see Table I). In order to address E-field dependence of the ZFS, we subsequently incorporate the $\mathrm{NV}^{-}$center into the (111) and (110) diamond slabs exposed to an electric field, represented by an extra sawtooth potential in the self-consistent cycle [43]. When the field is applied along the $\mathrm{NV}^{-}$defect axis, it acts on the zz-principal
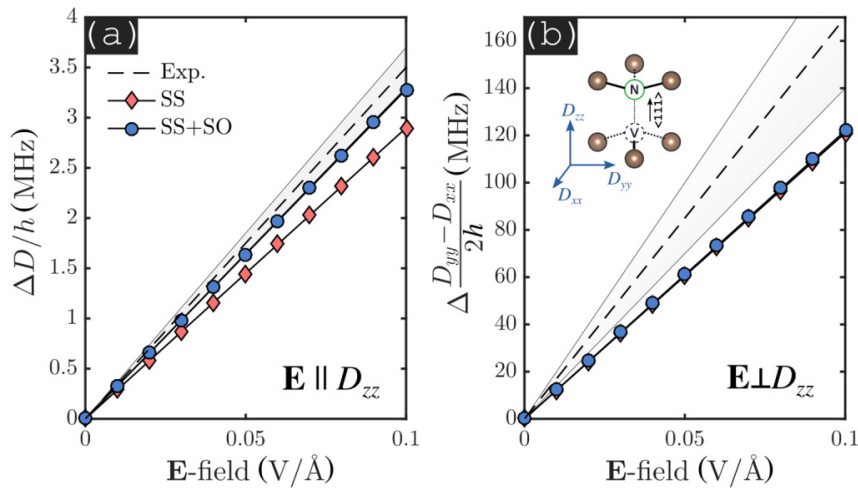

FIG. 2. Stark shifts of the principal values of the SS (red) and total $\left(\mathrm{SS}+\mathrm{SO}\right.$, blue) $\mathrm{ZFS}$ tensor of $\mathrm{NV}^{-}$in diamond. The results are shown for hydrogen-terminated 2D-periodic slab geometries with the E field parallel (a) and perpendicular (b) to the $D_{z z}$ principal direction. Experimental Stark coefficients [13] are shown with dashed lines and gray areas (experimental uncertainty interval).

components of the $\mathbf{D}^{\mathrm{SS}}$ and $\mathbf{D}^{\mathrm{SO}}$ tensors, i.e., directly on the $D$ value. An $\mathbf{E}$ field perpendicular to the defect's symmetry axis affects, instead, the difference of the $x x$ and $y y$ principal components [13], thereby introducing in-plane anisotropy of the ZFS tensor (see Fig. 2).

As expected from the minor SO contribution to the total ZFS of the $\mathrm{NV}^{-}$center (cf. Table I), the resulting Stark shift is predominantly caused by the spin-spin contribution reflecting the E-field-induced distortion of the magnetization density. For $\mathbf{E} \perp D_{z z}$, the influence of the SO part is even negligible [Fig. 2(b)], and the calculated Stark coefficient $1.221 \mathrm{GHz} \AA / \mathrm{V}$ agrees reasonably with the value of $1.70 \pm$ $0.25 \mathrm{GHz} \AA / \mathrm{V}$ estimated in Ref. [13] from an analysis of measured Hahn-echo decay curves. However, for the field applied along the defect's symmetry axis [Fig. 2(a)], the $D^{\mathrm{SO}}$ part tangibly drives the predicted Stark coefficient, $0.034 \mathrm{GHz}$ $\AA / \mathrm{V}$, into the experimentally observed confidence interval of $0.035 \pm 0.002 \mathrm{GHz} \AA / \mathrm{V}[13]$.

As a next step, we aim to systematically explore these effects for systems with larger SO couplings. Her we chose a sequence of group-IV impurity-decorated neutral divacancy centers (labeled $\mathrm{XV}^{0}$, where $\mathrm{X}$ denotes the $\mathrm{Si}, \mathrm{Ge}, \mathrm{Sn}$, or $\mathrm{Pb}$ impurity atom). The silicon- and germanium-vacancy centers were already addressed experimentally and reported to feature exceptional properties for optical initialization and readout [44-47]. The tin- and lead-vacancy defects were not yet observed in their neutral charge state but were successfully implanted with their negative charge state as potential singlephoton sources $[48,49]$.

The common $D_{3 d}$-symmetric structure of these $S=1$ defects is schematically illustrated in Fig. 3(a). Their two unpaired electrons are predominantly localized on the six carbon dangling bonds and not on the impurity atom itself [50]. Therefore, these defects exhibit almost identical magnetization density distribution and, consequently, similar $D^{\mathrm{SS}}$ values [Fig. 3(b)]. The SO-driven part, on the other hand, varies enormously with the impurity atom [Fig. 3(c)]. As follows from the analysis of the on-site PAW contributions [cf. Eq. (4)], a significant SO coupling stems from the spherical 

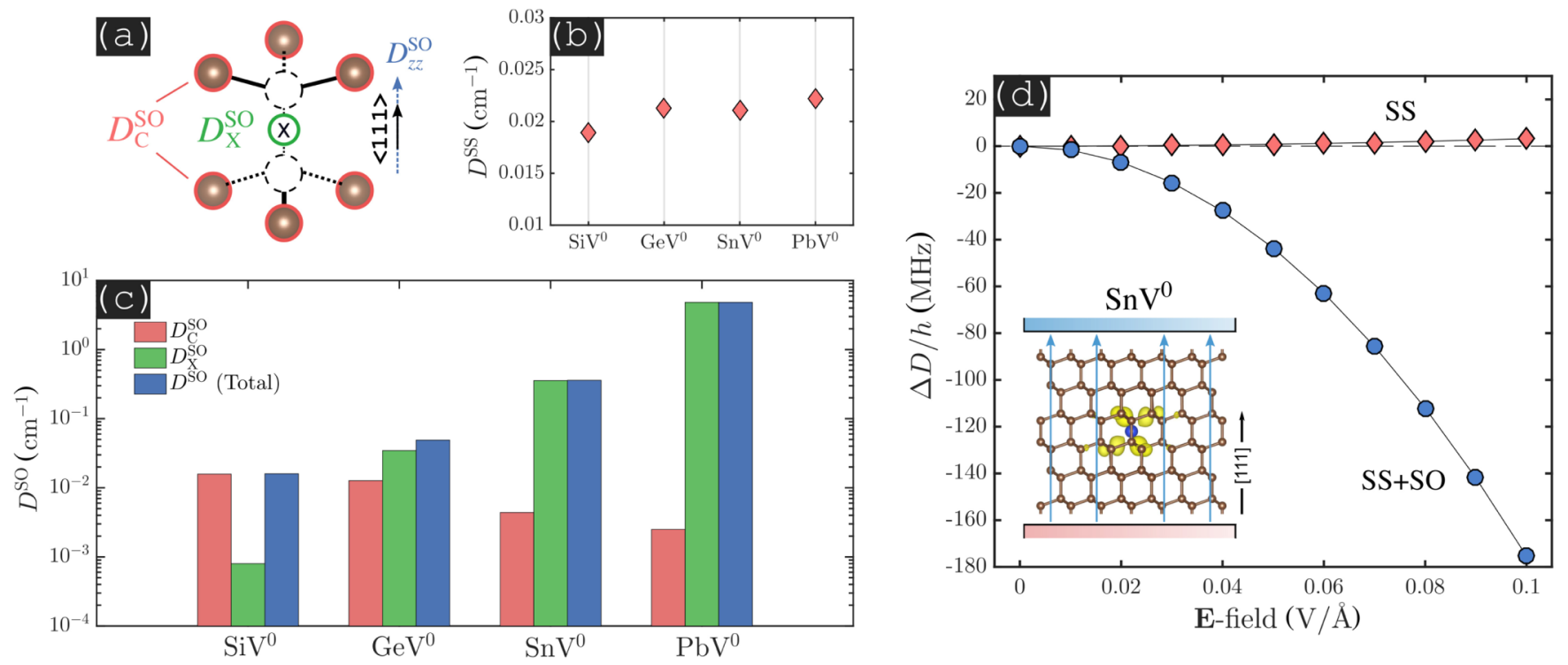

FIG. 3. Neutral impurity-vacancy centers in diamond ( $S=1$, axial symmetry): magnetic anisotropy (expressed by the $D$ value) projected onto atomic sites. (a) The impurity atom $\mathrm{X}$ occupies a central position within a divacancy (dashed circles). (b) The magnetization density (mainly localized in the $\mathrm{C}$ dangling bonds, see inset) gives rise to the spin-spin ZFS, $D^{\mathrm{SS}}$. (c) The impurity atom $\mathrm{X}$ and the $\mathrm{C}$ atoms of the host lattice contribute, respectively, with $D_{\mathrm{X}}^{\mathrm{SO}}$ and $D_{\mathrm{C}}^{\mathrm{SO}}$ to the total $D^{\mathrm{SO}}$. (d) E-field-induced shifts of the SO-driven (red diamonds) and total (SS $+\mathrm{SO}$, blue spheres) $D$ value of $\mathrm{SnV}^{0}$ calculated for a $2 \mathrm{D}$-periodic slab geometry (640 atoms, bottom inset).

regions around the host carbon atoms (a term denoted as $\left.D_{\mathrm{C}}^{\mathrm{SO}}\right)$. We correlate the enhanced carbon SO coupling with the local deformation of the diamond lattice (change of the C-C bond lengths $[51,52]$ ) induced by the impurity atoms. But most importantly, the impurity atom itself introduces the SO coupling $\left(D_{\mathrm{X}}^{\mathrm{SO}}\right)$ that becomes more and more dominant for heavier elements, i.e., with larger atomic SO coupling constants. Because of this, the overall SO-driven ZFS can become orders of magnitude larger than the spin-spin counterpart (Table I) and, thus, allows a sizable Stark shift. In Fig. 3(d) this is illustrated for $\mathrm{SnV}^{0}$. As expected for centers with inversion symmetry, there is no linear Stark effect and the induced shift in both $D^{\mathrm{SS}}$ and $D^{\mathrm{SO}}$ is thus quadratic. In contrast to the $\mathrm{NV}^{-}$center, the $\mathrm{SnV}^{0}$ defect's Stark coefficient of $17.6 \mathrm{GHz}(\mathrm{V} / \AA)^{-2}$ is caused almost entirely by the SO contribution.

\section{CONCLUSIONS}

In summary, the presented results enrich our understanding of SO physics of solid-state qubits. In particular, the SOdriven magnetic anisotropy is put forward as an essential lever for realizing future quantum applications of high-spin defects. We have provided a reliable second-order perturbative method to address the SO zero-field splitting (ZFS), which has not been previously accessible for extended periodic systems. The highly efficient pseudopotential approach allows us to describe systems with several hundreds or even thousands of atoms and provides all-electron accuracy. We find that both the spin-orbit and spin-spin parts of the ZFS respond to an external electric field. Even in light-element semiconductors, the Stark shift can be thus tuned in a broad range (1) by introducing a heavy impurity with large atomic SO coupling or (2) by a small impurity that induces the SO interaction via strong local strain. This profound insight paves the way for an electrically controlled tailoring of the magnetic anisotropy of high-spin defects suitable for quantum sensors and qubits.

\section{ACKNOWLEDGMENTS}

Numerical calculations were performed using grants of computer time from the Paderborn Center for Parallel Computing $\left(\mathrm{PC}^{2}\right)$. The Deutsche Forschungsgemeinschaft (DFG) is acknowledged for financial support via the priority program SPP1601.
[1] J. R. Weber, W. F. Koehl, J. B. Varley, A. Janotti, B. B. Buckley, C. G. Van de Walle, and D. D. Awschalom, Proc. Natl. Acad. Sci. USA 107, 8513 (2010).

[2] B. E. Kane, Nature (London) 393, 133 (1998).

[3] R. Rahman, C. J. Wellard, F. R. Bradbury, M. Prada, J. H. Cole, G. Klimeck, and L. C. L. Hollenberg, Phys. Rev. Lett. 99, 036403 (2007).
[4] K. C. Nowack, F. H. L. Koppens, Y. V. Nazarov, and L. M. K. Vandersypen, Science 318, 1430 (2007).

[5] A. Laucht, J. T. Muhonen, F. A. Mohiyaddin, R. Kalra, J. P. Dehollain, S. Freer, F. E. Hudson, M. Veldhorst, R. Rahman, G. Klimeck, and K. M. Itoh, Sci. Adv. 1, e1500022 (2015).

[6] S. Nadj-Perge, S. M. Frolov, E. P. A. M. Bakkers, and L. P. Kouwenhoven, Nature (London) 468, 1084 (2010). 
[7] A. Crippa, R. Maurand, L. Bourdet, D. Kotekar-Patil, A. Amisse, X. Jehl, M. Sanquer, R. Laviéville, H. Bohuslavskyi, L. Hutin, S. Barraud, M. Vinet, Y.-M. Niquet, and S. De Franceschi, Phys. Rev. Lett. 120, 137702 (2018).

[8] G. Wolfowicz, M. Urdampilleta, M. L. W. Thewalt, H. Riemann, N. V. Abrosimov, P. Becker, H. J. Pohl, and J. J. L. Morton, Phys. Rev. Lett. 113, 157601 (2014).

[9] A. Abragam and B. Bleaney, Electron Paramagnetic Resonance of Transition Ions (Clarendon, Oxford, 1970).

[10] D. Gatteschi and R. Sessoli, Angew. Chem. Intl. Ed. 42, 268 (2003).

[11] R. E. George, J. P. Edwards, and A. Ardavan, Phys. Rev. Lett. 110, 027601 (2013).

[12] A. Kiel and W. B. Mims, Phys. Rev. B 5, 803 (1972).

[13] E. van Oort and M. Glasbeek, Chem. Phys. Lett. 168, 529 (1990).

[14] F. Dolde, H. Fedder, M. W. Doherty, T. Nöbauer, F. Rempp, G. Balasubramanian, T. Wolf, F. Reinhard, L. C. Hollenberg, F. Jelezko, and J. Wrachtrup, Nat. Phys. 7, 459 (2011).

[15] F. Dolde, M. W. Doherty, J. Michl, I. Jakobi, B. Naydenov, S. Pezzagna, J. Meijer, P. Neumann, F. Jelezko, N. B. Manson, and J. Wrachtrup, Phys. Rev. Lett. 112, 097603 (2014).

[16] Y. A. Bychkov and E. I. Rashba, J. Phys. C 17, 6039 (1984).

[17] J. Nitta, T. Akazaki, H. Takayanagi, and T. Enoki, Phys. Rev. Lett. 78, 1335 (1997).

[18] M. J. Rayson and P. R. Briddon, Phys. Rev. B 77, 035119 (2008).

[19] T. Biktagirov, W. G. Schmidt, and U. Gerstmann, Phys. Rev. B 97, 115135 (2018).

[20] T. Biktagirov, W. G. Schmidt, and U. Gerstmann, arXiv:1909.13577 [Phys. Rev. Research (to be published)].

[21] Z. Bodrog and A. Gali, J. Phy.: Condens. Matter 26, 015305 (2013).

[22] H. Seo, H. Ma, M. Govoni, and G. Galli, Phys. Rev. Mater. 1, 075002 (2017).

[23] F. Neese, J. Chem Phys. 127, 164112 (2007).

[24] M. Atanasov, D. Aravena, E. Suturina, E. Bill, D. Maganas, and F. Neese, Coord. Chem. Rev. 289-290, 177 (2015).

[25] C. Duboc, Chem. Soc. Rev. 45, 5834 (2016).

[26] Y. Rechkemmer, F. D. Breitgoff, M. van der Meer, M. Atanasov, M. Hakl, M. Orlita, P. Neugebauer, F. Neese, B. Sarkar, and J. van Slageren, Nat. Commun. 7, 10467 (2016).

[27] P. E. Blöchl, Phys. Rev. B 50, 17953 (1994).

[28] U. Gerstmann, N. J. Vollmers, A. Lücke, M. Babilon, and W. G. Schmidt, Phys. Rev. B 89, 165431 (2014).

[29] P. Giannozzi, O. Andreussi, T. Brumme, O. Bunau et al., J. Phys.: Condens. Matter 29, 465901 (2017).
[30] P. Giannozzi, S. Baroni, N. Bonini, M. Calandra et al., J. Phys.: Condens. Matter 21, 395502 (2009).

[31] J. P. Perdew, K. Burke, and M. Ernzerhof, Phys. Rev. Lett. 77, 3865 (1996).

[32] N. Troullier and J. L. Martins, Phys. Rev. B 43, 1993 (1991).

[33] P. Bruno, Phys. Rev. B 39, 865 (1989).

[34] D. S. Wang, R. Wu, and A. J. Freeman, Phys. Rev. B 47, 14932 (1993).

[35] M. R. Pederson and S. N. Khanna, Phys. Rev. B 60, 9566 (1999).

[36] D. Ceresoli, U. Gerstmann, A. P. Seitsonen, and F. Mauri, Phys. Rev. B 81, 060409(R) (2010).

[37] C. J. Pickard and F. Mauri, Phys. Rev. Lett. 88, 086403 (2002).

[38] C. J. Pickard and F. Mauri, Phys. Rev. B 63, 245101 (2001).

[39] Indeed, its elements, $D_{a b}^{\mathrm{SO}}$, are built by multiplying $E_{a b}^{\mathrm{SO}}$ by the coefficient from Eq. (3) and, conventionally, by subtracting the trace, which describes collective shift of the spin sublevels (see also Ref. [9] for details).

[40] F. Neese, Wiley Interdiscip. Rev. Comp. Mol. Sci. 2, 73 (2012).

[41] W. Florian and R. Ahlrichs, Phys. Chem. Chem. Phys. 7, 3297 (2005).

[42] J. Loubser and J. van Wyk, Rep. Prog. Phys. 41, 1201 (1978).

[43] S. Müllegger, E. Rauls, U. Gerstmann, S. Tebi, G. Serrano, S. Wiespointner-Baumgarthuber, W. G. Schmidt, and R. Koch, Phys. Rev. B 92, 220418(R) (2015).

[44] K. Iakoubovskii and A. Stesmans, Phys. Rev. B 66, 195207 (2002).

[45] A. M. Edmonds, M. E. Newton, P. M. Martineau, D. J. Twitchen, and S. D. Williams, Phys. Rev. B 77, 245205 (2008).

[46] B. C. Rose, G. Thiering, A. M. Tyryshkin, A. M. Edmonds, M. L. Markham, A. Gali, S. A. Lyon, and N. P. de Leon, Phys. Rev. B 98, 235140 (2018).

[47] V. Nadolinny, A. Komarovskikh, Y. Palyanov, I. Kupriyanov, Y. Borzdov, M. Rakhmanova, O. Yuryeva, and S. Veber, Phys. Stat. Sol. (A) 213, 2623 (2016).

[48] M. E. Trusheim, N. H. Wan, K. C. Chen, C. J. Ciccarino, J. Flick, R. Sundararaman, G. Malladi, E. Bersin, M. Walsh, B. Lienhard, H. Bakhru, P. Narang, and D. Englund, Phys. Rev. B 99, 075430 (2019).

[49] T. Iwasaki, Y. Miyamoto, T. Taniguchi, P. Siyushev, M. H. Metsch, F. Jelezko, and M. Hatano, Phys. Rev. Lett. 119, 253601 (2017).

[50] A. Gali and J. R. Maze, Phys. Rev. B 88, 235205 (2013).

[51] A. H. Castro Neto and F. Guinea, Phys. Rev. Lett. 103, 026804 (2009).

[52] U. Gerstmann, A. P. Seitsonen, D. Ceresoli, F. Mauri, H. J. von Bardeleben, J. L. Cantin, and J. Garcia Lopez, Phys. Rev. B 81, 195208 (2010). 